\title{
SINGLE-STEP RENAL DILATATION IS A NOVEL TECHNIQUE IN PERCUTANEOUS NEPHROLITHOTOMY: A PROSPECTIVE RANDOMISED STUDY
}

\section{SHARIFUL ISLAM ${ }^{1}$, MD. SHAWKAT ALAM ${ }^{2}$, A.H.M MOSTOFAKAMAL ${ }^{2}$, MD. ASADUZZAMMAN ${ }^{3}$, MD.RUHUL QUDDUS ${ }^{4}$}

${ }^{1}$ Department of Urology, STAMC, Gajipur, ${ }^{2}$ Department of urology, NIKDU, ${ }^{3}$ Department of urology, Dinajpur medical college, ${ }^{4}$ Department of Surgery, Satkhira Medical College.

\begin{abstract}
Purpose: To evaluate the feasibility, safety and efficacy of one-shot dilation (OSD) in percutaneous nephrolithotomy (PCNL).

Materials and Methods: In a prospective randomised study including 62 patients divided into two groups, the first had RD before PCNL using the standard metallic telescopic dilators (Alken), and the second had $R D$ using the 30-F Amplatz dilator over the central Alken dilator. The operative duration, with $X$-ray exposure, was calculated. The procedure outcome in terms of complications, stone-free rates and hospital stay was evaluated statistically.

Results : The tract was dilated correctly in all cases. The operative duration and X-ray exposure was shorter in patients undergoing single-step $R D(P<0.05)$. There were perioperative complications, according to the Clavien grading system, in $15(25 \%)$ patients but there was no statistically significant difference between the groups. The stone-free rates were comparable in both groups.

Conclusion : A single-step RD during PCNL is feasible, with a shorter operative duration and $X$-ray exposure. The outcomes were comparable with those of a standard metallic telescopic RD
\end{abstract}

Key words Renal dilatation;Single-step;Fascial dilators;Telescopic dilator

Bangladesh J. Urol. 2017; 20(2): 70-73

\section{Introduction}

Renal stones represents a large portion in the field of urological pathology. Percutaneous nephrolithotomy $(\mathrm{PCNL})$ is the gold standard procedure for treating renal stones larger than $2 \mathrm{~cm}$. PCNL has replaced open surgical removal of large or complex calculi at the most institutions This technique is associated with high success rates, decreased morbidity and fewer complications than conventional renalstone surgery [1]. An important and integral step in PCNL is renal puncture and tract dilatation with insertion of the access sheath, but during these steps bleeding can occur, although it usually responds to the tamponade effect provided by the access sheath [2].

Correspondences: Md. Shariful Islam, Department of Urology, STAMC,Gajipur,
One of the most fundamental steps of percutaneous nephrolithotomy (PCNL) is the creation of the nephrostomy access. It can be done with serial polyurethane co-axial dilators (Amplatz dilators), balloon dilators and telescoping metallic co-axial dilators (Alken dilators). Use of an Amplatz dilator set or metal incremental dilators is time consuming and requires longer exposure to fluoroscopy.[1]

More recently, to reduce access time and radiation exposure during access, and to reduce cost, a singlestep technique involving the use of an Amplatz serial dilator over a metallic telescopic dilator ("one-shot") has become accepted as a safe and effective technique.[1-3]

Metal telescopic dilators are reusable and thus more economical, and they maintain a tamponade effect 
throughout the dilatation. Previous reports suggest no difference between metallic and sequential fascial dilatation in terms of safety and efficiency [3]. Fascial dilators are disposable and it has been suggested that during sequential dilator exchanges, the tamponade effect on the renal parenchymal tract is lost, which can lead to more blood loss during the exchange process [4]. Both metallic and sequential fascial dilators are timeconsuming to use, with an increased incidence of kinking of the guidewire during tract development, thus hindering adequate RD.

Many studies have been performed to study the damage caused by tract dilatation during PCNL. The use of multiincrement dilators was time-consuming and with each pass of the dilator cause injury to the collecting system .Also, there is a risk of significant bleeding when a sequential dilator is removed to allow placement of the larger one, because tract is left open, and there is no sheath in place to tamponed the bleeding. For this, some authors proposed single-increment dilation and demonstrated its safety and feasibility. Travis and colleagues, 1991 proved that single-increment dilatation was as safe as conventional techniques with minimal haemorrhage or parenchymal damage and healing at 6 weeks by a fine linear scar. Frattini and colleagues,2001 who first described the one shot dilation(OSD) technique and found a reduction in the fluoroscopy time for tract dilation than conventional dilation technique and thereby got advantages of less radiation exposure to both urologist and patient, and shorter access and operative time. They also observed that OSD lead to less haemorrhagic complications than multi-incremental dilation technique. In two others studies, Falahatkar et al, 2009 and Amjadi et al, 2008 OSD and telescopic dilation had a similar haematologic safety profiles.

Thus in this study we assessed the use of an economical single-step RD using directly the 26-F Amplatz dilator over the central Alken dilator in a trial to reduce the operative duration and radiation exposure during RD, while avoiding an exchange of dilators that might increase the risk of blood loss.

\section{Patients and Methods}

This hospital based prospective comparative study was conducted on the patients with renal stone $(\geq 2 \mathrm{~cm})$, admitted in the department of Urology, NIKDU, Dhaka from July 2014 to June 2016 to compare the outcome of single-step dilatation and multi-increment dilatation technique for PCNL. Decision of operation was taken as per the standard protocol of the urology department of NIKDU.
All patients was evaluated by detailed history, physical examination and some investigations. Urinalysis, urine culture and sensitivity, complete blood count (CBC), blood urea nitrogen (BUN), serum creatinine, coagulation profile and plain X-Ray KUB region, ultrasonography of KUB region, IVU or Non contrast CT Scan of KUB region were done. Patient with documented urinary tract infection was treated with appropriate antibiotic before surgery.

Patients selected for PCNL in OPD were then to be admitted in different Urology units in NIKDU should undergo surgery. PCNL was carried out, in half of the patients tract dilation was done by one shot technique and in rest half of patients tract dilation was done by multi-increment technique. Cystoscopy was done with insertion of an open-tip 7- F ureteric catheter, followed by renal puncture in the prone position and insertion of both the first and safety guidewire. The lumbodorsal fascia was punctured and split using a long haemostat under fluoroscopy (Fig. 1). The central Alken dilator was advanced over the guidewire and this was followed by directly inserting the 30-F Amplatz dilator, with the surgeon applying constant pressure and slowly advancing the dilator and then the access sheath under fluoroscopy (Fig. 2). We then used a 24-F nephroscope with a ballistic energy source for stone disintegration. Per operative efforts, haemorrhage, stone clearance and total operating time will be noted carefully.

The data obtained were assessed statistically, with $\mathrm{P}<$ 0.05 considered to indicate statistical significance.

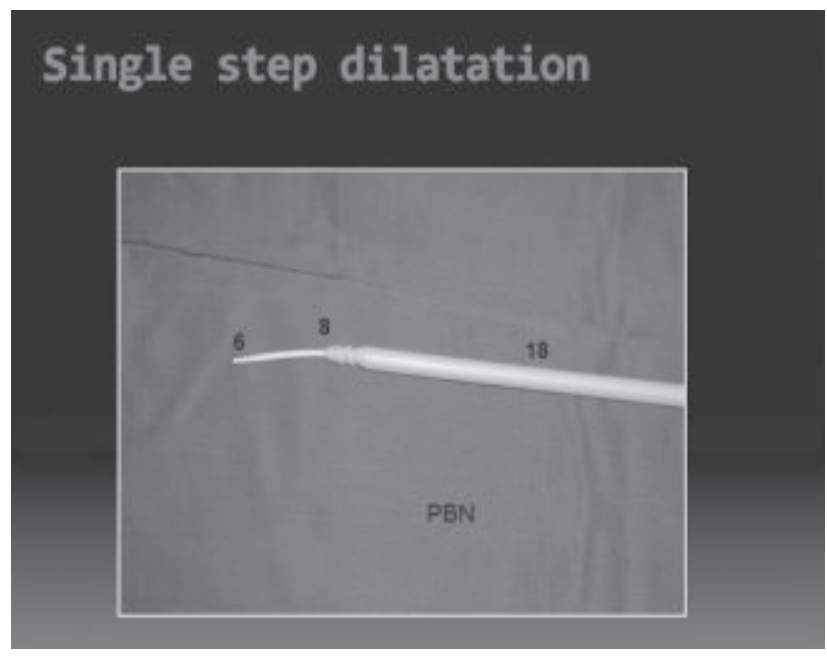

Fig.-1 


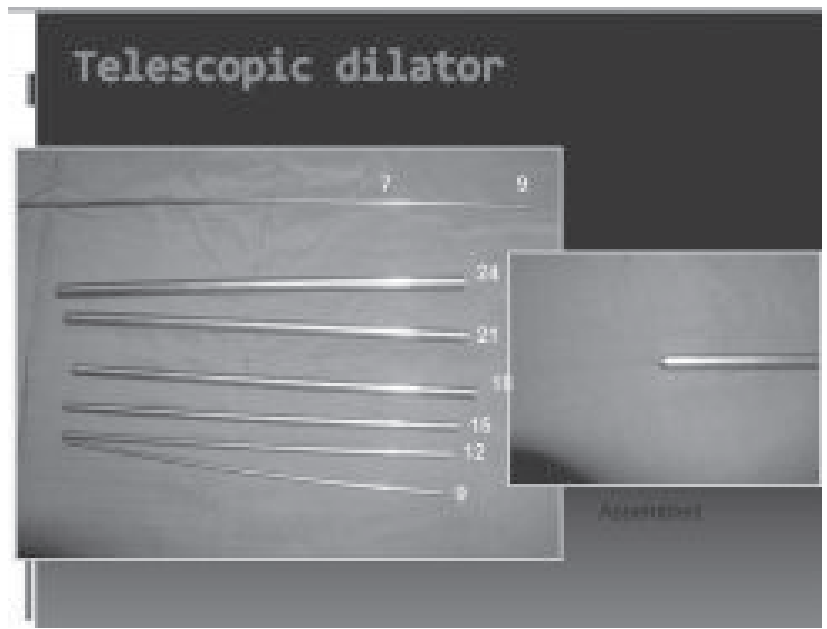

Fig.2

\section{Results}

Of 60 cases, Patients having tract dilatation using the single-step technique were statistically significantly older than those having their tract dilated using the Alken dilators; otherwise,there were no differences in the clinical variables between the groups (Table I).

Table- I

\begin{tabular}{lcc}
\hline Variable & One shot dilatation & $\begin{array}{c}\text { Sequential } \\
\text { dilation }\end{array}$ \\
\hline No. of pt. & 30 & 30 \\
age & $38.2 \pm 12.5$ & $42.8 \pm 13.4$ \\
Sex(m/f) & $20 / 10$ & $18 / 12$ \\
Stone burden & $30.2 \pm 6.2$ & $30.5 \pm 7.2$ \\
Preop. HB\% & $13.3 \pm 1.7$ & $13.2 \pm 1.5$ \\
OT time & $99.7 \pm 25.1$ & $124.6 \pm 23$ \\
Fluoroscopy time & $10.5 \pm 0.6$ & $14.4 \pm 0.6$ \\
Failure of acess & 0 & 0 \\
Stone clerarance & $27(90 \%)$ & $28(93.3 \%)$ \\
Post. Op. HB\% & $12.2 \pm 0.7$ & $11.5 \pm 0.5$ \\
complications & 7 & 8 \\
Transfusion rate & 1 & 2 \\
Organ injury & 0 & 0 \\
Hospital stay & $4.1 \pm 1$ & $4.8 \pm 1.1$ \\
\hline
\end{tabular}

In all patients there was renal access with correct tract dilatation, and the operative duration and that of fluoroscopy exposure was statistically significantly longer when using the Alken dilators than with the singlestep RD (Table I). The stone-free rate was assessed using a plain abdominal film on the day after surgery, with residual fragments of $<5 \mathrm{~mm}$ considered to be clinically insignificant. There were residual fragments in three $(5 \%)$ of the 60 patients. There was no significant difference in stone-free rates between the groups. There was no statistically significant blood loss (comparing haemoglobin levels before and after surgery) in both groups, or between the postoperative values in both groups. A blood transfusion was needed in two patients, one from each group.

There were complications, according to the modified Clavien grading system, in 15 (25\%) patients; six (40\%) had grade 1 complications, with grade 2 and 3 complications in six $(40 \%)$ and three $(20 \%)$, respectively. There was no statistically significant difference in complication rates between the groups, and no reported organ injury in any patient.

In the absence of complications, patients were discharged on the same day the urinary catheter was removed, and there was no statistically significant difference in the duration of hospital stay between the groups (Table I).

\section{Discussion}

Tract dilatation is an essential step in PCNL, and inadequate $\mathrm{RD}$ can hinder the introduction of the access sheath and provoke bleeding. RD is classically done using metallic telescopic dilators (Alken), sequential fascial dilators (Amplatz), and single-step balloon dilator. Although all three methods are usually safe, reports show a higher blood loss for metallic telescopic RD than for the Amplatz and balloon dilatation [6].

The Clinical Research Office of the Endourology Society (CROES) has recently released the results of a global PCNL study involving 5803 patients in 96 centres around the world. The observational analysis found that ASD was associated with lower rates of haemorrhage $(6.7 \%$ v. $9.4 \%)$ and a lower transfusion rate $(4.9 \%$ v. $7 \%)$ when compared with metallic telescopic \& also balloon dilatation devices. Alimitation of the study was that intercentre indications for PCNL varied, with a larger number of staghorn calculi being treated in the balloon dilatation group.

In the present study the duration of X-ray exposure was statistically lower for single-step Amplatz RD than for metallic RD. A shorter $X$-ray exposure was also reported by Frattini et al. [7], who used one-step RD with a 25$\mathrm{F}$ or 30-F Amplatz dilator to gain renal access.

In the present study the overall operative duration when using the single-step Amplatz RD was significantly 
shorter than when using Alken dilators. The single-step Amplatz RD was safe and effective [10], and in the present study the stone-free rates were comparable in both groups, as were the complication rates, which also did not differ between the groups. Although not objectively measured nor compared statistically, the cost of using the reusable telescopic metallic dilators (Alken) and the single-step 30-FAmplatz dilator is apparently lower than for the nonreusable balloon dilator.

The present study had several limitations, as it

included relatively few patients, did not consider the effect of previous renal surgery, and did not test the efficiency of this technique when supracostal renal access was used

In conclusion, the single-step Amplatz RD is a timesaving procedure, being safe, subjectively economical and an effective technique to gain renal access. We found no specific complication with this technique, and the morbidity rates were comparable with those of the classical metallic telescopic $\mathrm{RD}$. We recommend its use for patients undergoing PCNL.

\section{References}

1. Bird VG, Fallon B, Winfield HN. Practice patterns in the treatment of large renal stones. J Endourol 2003;17:355-63.

2. Ganpule AP, Shah DH, Desai MR. Postpercutaneous nephrolithotomy bleeding aetiology and management. Curr Opin Urol 2014;24L:189-94.

3. Ozok HU, Sagnak L, SenturkAB, Karakoyunlu N, Topaloglu H, Ersoy H. A comparison of metal telescopic dilators and Amplatz dilators for nephrostomy tract dilation in percutaneous nephrolithotomy. J Endourol 2012;26:630-4.

4. Yuhico MP, Ko R. The current status of percutaneous nephrolithotomy in the management of kidney stones. Minerva Urol Nefrol 2008;60:159_ 75.

5. Lee KL, Stoller ML. Minimizing and managing bleeding after percutaneous nephrolithotomy. Curr Opin Urol 2007;17:120-4.

6. Dehong C, Liangren L, Huawei L, Qiang WA. Comparison among four tract dilation methods of percutaneous nephrolithotomy: a systematic review and meta-analysis. Urolithiasis 2013;41:523-30.

7. Frattini A, Barbieri A, Salsi P, Sebastio N, Ferretti $S$, Bergamaschi E, et al. One shot. A novel method to dilate the nephrostomy access for percutaneous lithotripsy. J Endourol 2001;15:919-23.

8. Falahatkar S, Neiroomand H, Akbarpour M, Emadi SA, Khaki,N. One-shot versus metal telescopic dilation technique for tract creation in percutaneous nephrolithotomy. Comparison of safety and efficacy. J Endourol 2009;23:615-8.

9. Amjadi M, Zolfaghari A, Elahian A, Tavoosi A. Percutaneous nephrolithotomy in patients with previous open nephrolithotomy: one-shot versus telescopic technique for tract dilatation. J Endourol 2008;22:423-5.

10. Amirhassani S,Mousavi-Bahar SH, lloon Kashkouli A, Torabian S. Comparison of the safety and efficacy of one-shot and telescopic metal dilatation in percutaneous nephrolithotomy: a randomized controlled trial. Urolithiasis 2014;42:269-73. 